\title{
Site-specific lift out sample preparation technique for atom probe analysis
}

\author{
D. Lawrence*, K. Thompson*, D. Larson*, B Gorman** \\ *Imago Scientific Instruments Corp. 6300 Enterprise Ln, Suite 100, Madison, WI 53719 \\ ** University of North Texas. P.O. Box 305310, Denton, Texas, 76203-5310
}

Atom-probe analysis of blanket Si wafers has demonstrated the potential of atom-probe tomography as a characterization tool for Si based nanostructures [1]. The challenge now is not the atom-probe analysis but instead the ability to make an appropriate atom-probe specimen from a specific structure located in a full-flow device wafer. Borrowing on the tools and techniques available for work done on site-specific TEM sample preparation $[2,3,4]$, a systematic approach to site-specific atom-probe specimen preparation has been developed.

The sample for analysis and a LEAP ${ }^{\circledR}$ microtip array are loaded into an FEI Nova 235 Nanolab dualbeam SEM/FIB system with a Magnum ion column. A $150 \mathrm{~nm}$ thick protective platinum bar, which completely covers and protects the area to be lifted out, is deposited in a rectangular pattern: 2 microns wide by 50 microns long. The stage is tilted to 22 degrees relative to the electron beam such that a 30 degree cut is made in the wafer just below the region protected by the Pt bar. The cut pattern is 0.5 microns wide, 50 microns long and 5 microns deep. A high beam current $(\sim 7 \mathrm{nA})$ is utilized to minimize milling time. The wafer is then rotated by 180 degrees relative to the ion column and the cut step repeated on the other side of the Pt bar. The result is a wedge shaped structure still attached to the substrate at either end. Subsequent shorter duration clean-up cuts may be made to remove any re-deposition.

Tilting the stage back to zero degrees, the omniprobe micromanipulator and platinum deposition systems are utilized to extract the wedge. The micromanipulator is brought into intimate contact with one end of the wedge, Fig. 1, and attached via Pt deposition. Cuts are made on either side of the wedge to free it from the wafer, and the manipulator is retracted to extract the sample wedge, Fig. 2. The lift-out is then translated to the desired mounting fixture, in this case the 100 micron tall silicon posts that compose the LEAP microtip array, Figs. 3 and 4. The bottom of the wedge is aligned to the top of the post and attached by Pt deposition in the open area between the sample wedge and the post, Fig. 5. The wedge is then sliced away, Fig. 6, leaving the sample attached to the top of the microtip post. The wedge is then retracted and this mount and slice process repeated until the entire wedge is consumed. It is reasonable to expect $\sim 18$ samples from a 50 micron long wedge. Once the slice is consumed, the LEAP microtip array is rotated by 180 degrees and the back-side of the sample is filled with Pt to improve the stability of the mounted sample.

The mounted sample is milled using annular patterns, resulting in an atom probe sample of appropriate tip radius and shank angle, Figs. 7 and 8. The final step is the application of a low keV clean-up mill (i.e. 2 or $5 \mathrm{keV}$ beam) to remove the excess Pt cap and the Ga damaged regions [5].

[1] K.Thompson, J.H. Booske, D.J. Larson, T.F. Kelly, Appl. Phys. Lett. 87(5) 2005.

[2] L.A. Giannuzzi, R. Geurts, J. Ringnalda, Microscopy \& Microanalysis 1192 (2005) 828.

[3] D.J. Larson, et al, Microscopy and Microanalysis 7, (2001) 24.

[4] M.K. Miller, et al, Strategies for Fabricating Atom Probe Specimens with a Dual Beam FIB Ultramicroscopy, 102 (2005) 287-298

[5] K. Thompson, B. Gorman, D. J. Larson, B. van Leer, L. Hong, "Minimization of Ga Induced FIB Damage Using Low Energy Clean-up" this Proceeding. 

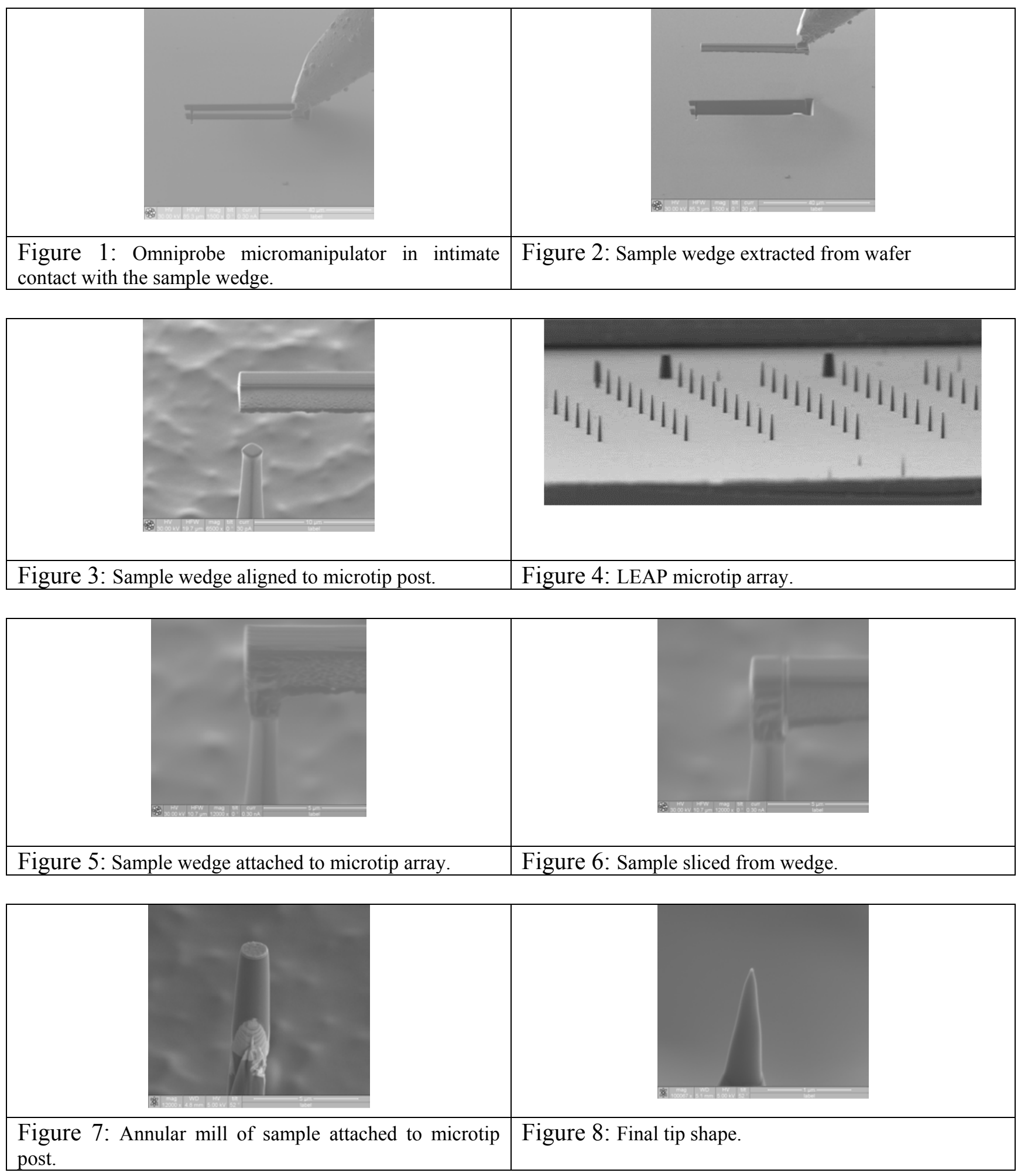